\title{
CONSTRUCCIÓN DEL MODELO EDUCATIVO DE UNA UNIVERSIDAD GUATEMALTECA CON CRITERIOS DE INTERNACIONALIZACIÓN CURRICULAR
}

\author{
CONSTRUCTION OF THE EDUCATIONAL MODEL OF A GUATEMALAN \\ UNIVERSITY WITH CURRICULAR INTERNATIONALIZATION CRITERIA
}

\author{
René Cristóbal CROCKER SAGASTUME ${ }^{1}$ \\ Regina del Carmen SAGASTUME LIZAMA²

\begin{tabular}{|lll|}
\hline Recibido & $:$ & 02.04 .2021 \\
Aceptado & $:$ & 26.07 .2021 \\
Publicado & $:$ & 02.08 .2021 \\
\hline
\end{tabular}

RESUMEN: Se analiza la práctica educativa para construir el modelo educativo de la Universidad de San Carlos de Guatemala (USAC) a partir de la experiencia del Centro Universitario de Ciencias de la Universidad de Guadalajara, donde se desarrolla un currículo basado en el enfoque de competencias profesionales integradas con el objetivo de mostrar la importancia del papel de la internacionalización curricular como un elemento de colaboración entre universidades públicas de América Latina. Se aplica un diseño metodológico de investigación acción participativa en tres fases: formación de los académicos en las dimensiones del modelo educativo, diagnóstico situacional de la problemática de desarrollo curricular de la institución y construcción del modelo educativo. Se elabora una propuesta fundamentada en el paradigma socio-histórico cultural, que incluye cuatro dimensiones: Filosófica, administrativo-académica, pedagógicodidáctica y gestión de la calidad educativa, que es discutida en las áreas académicas. Se concluye, que el éxito de los procesos de internacionalización del desarrollo curricular está relacionado con la firma de convenios previos, la sistematización de las propuestas propias, la gestión interinstitucional de largo plazo, la aplicación de metodologías participativas para el desarrollo curricular con los miembros de las instituciones vinculadas y el compromiso de los asesores que participen en procesos de internacionalización.

Palabras clave: Internacionalización, currículo, modelo educativo, universidades públicas, práctica educativa.

\begin{abstract}
The educational practice is analyzed to construct the educational model of University of San Carlos, Guatemala (USAC) from the experience of University Science Center of the University of Guadalajara, where a curriculum based on an integrated professional competences approach is developed with the aim of showing the importance of the role of curricular internationalization as an element of collaboration between public universities in Latin America. A methodological design of participatory action research is applied in three phases: training of academics in the dimensions of the educational model, situational diagnosis of problem of curricular development of the institution and construction of the educational model. A proposal based on the socio-historical-cultural paradigm is elaborated, which includes four dimensions: philosophical, administrative-academic, pedagogical-didactic and educational quality management, discussed in the academic areas. It is concluded that the success of the internationalization processes of the curricular development is related to the signing of previous agreements, the systematization of the proposals, the long-term inter-institutional management, the application of participatory methodologies for the curriculum development with the members of the linked institutions and the commitment of the advisers who participate in processes of internationalization.
\end{abstract}

Keywords: Curriculum, Educational Model, Educational Practice, Internationalization, Public Universities.

\footnotetext{
${ }^{1}$ Médico y Cirujano, Dr. en Investigación Educativa Aplicada. Profesor Investigador Titular del Instituto Regional de Investigación en Salud Pública. Universidad de Guadalajara, Guadalajara, Jalisco México. Email: recricrosa_7@ hotmail.com. ORCID: https://orcid.org/0000-0001-9425-2126

${ }^{2}$ Médica y Cirujana, Maestría en Medición, Evaluación e Investigación Educativa. Asesora de la Coordinadora de Planificación de la Facultad de Ciencias Médicas. Universidad de San Carlos de Guatemala, Guatemala. Email: regina.sagastume@gmail.com. ORCID: https://orcid.org/0000-0002-0047-7058
} 


\section{INTRODUCCIÓN}

Se analiza la práctica educativa para construir el modelo educativo de la Universidad de San Carlos de Guatemala (USAC) con base en el intercambio de experiencias con el Centro Universitario de Ciencias de la Universidad de Guadalajara, donde se desarrolla un currículo basado en el Enfoque de Competencias Profesionales Integradas (Crocker et al., 2008) con el objetivo de mostrar la importancia del papel de la internacionalización curricular como un elemento de colaboración entre universidades públicas de América Latina.

Se documenta la vinculación que ha desarrollado la Universidad de Guadalajara, a través de investigadores en el campo de la educación del Centro Universitario de Ciencias de la Salud, con la USAC, desde el año 2005 (Universidad de Guadalajara /USAC, 2005) y que incluye en esta última etapa, una consultoría realizada por el coordinador de la investigación en los años 2017 a 2019, con el equipo de la Dirección General de Desarrollo Académico (DIGED) de la USAC, para responder a la petición de la Facultad de Ciencias Médicas de dicha institución educativa de Guatemala, que demanda la construcción del Modelo Educativo Institucional, que no existía de manera formal, como parte de los indicadores de calidad relacionados con la acreditación de carreras universitarias formulados por el Sistema Centroamericano de Evaluación y Armonización de la Educación Superior. (Martinez, 2005)

Los fundamentos de la internacionalización curricular de la USAC, han sido construidos en el Centro Universitario de Ciencias de la Salud de la Universidad de Guadalajara. El coordinador del proceso de internacionalización curricular, forma parte de un equipo de investigadores educativos en el campo de las ciencias de la salud de la Universidad de Guadalajara, que han venido liderando la innovación curricular con el Enfoque de Competencias Profesionales Integradas desde el año 2005. Para fundamentar la innovación curricular se construyó el Modelo Educativo del Centro Universitario de Ciencias de la Salud (CUCS) (Crocker et al., 2009a) El proceso de innovación curricular con el Enfoque de Competencias Profesionales Integradas, fue evaluado por la Comisión Base de Desarrollo Curricular del CUCS, en el año 2010, con el propósito de mejorar e innovar la calidad educativa a escala nacional e internacional. (Farfán et al., 2010) 


\section{Journal of the Academy $|158|$}

La estrategia de construcción del modelo educativo, surge de la necesidad de acreditación de la Unidad Académica Facultad de Ciencias Médicas, donde se deriva la construcción de un modelo académico general de la USAC. Al analizar el contexto institucional en la Facultad de Ciencias Médicas, como un espacio representativo para el desarrollo del modelo educativo de la USAC, los autores en diálogo con el equipo coordinador de la institución identifican los siguientes elementos:

a) Elementos contextuales: El contexto donde se desarrolla la sociedad guatemalteca está determinado por la Globalización Neoliberal y la Tercera Revolución Científicotecnológica que ha provocado una polarización social y demográfica, lo que constituye un reto para la construcción del modelo educativo de la USAC.

b) Elementos educativos: El papel de liderazgo que juega la USAC en la educación superior en Guatemala, demanda realizar análisis educativos sustentados en enfoques complejos que incluyan, entre otros elementos, el análisis de la realidad social, la competitividad de los egresados en los mercados laborales y en el contexto global, sin dejar de lado, la formación humano social y aspectos relacionados con la identidad profesional (Dirección General de Desarrollo Académico USAC, 2018)

Para analizar el proceso y desarrollar el modelo educativo de la USAC que contribuya a la acreditación de la Facultad de Ciencias Médicas, se formula las siguientes preguntas de investigación educativa:

1. ¿Cuáles elementos del contexto externo e interno se deben valorar por los actores de la USAC para formular un modelo educativo con el enfoque de competencias?

2. ¿Cuál es la problemática curricular, pedagógico-didáctica y de profesionalización docente que deberá ser evaluada para implementar un modelo educativo por competencias?

3. ¿Cuáles son los problemas que enfrentan las unidades académicas de la USAC para su acreditación en los organismos regionales e internacionales?

La experiencia que se documenta tiene como antecedente la vinculación que ha establecido desde el año 2005 la Universidad de Guadalajara en el marco del Programa de Intercambio Académico de la Asociación Nacional de Universidades e Instituciones de Educación Superior 


\section{Journal of the Academy $|159|$}

(ANUIES) con el Consejo Superior de Universidades de Centro América (CSUCA) en donde el coordinador de la investigación curricular, se incorpora para promover la firma del Convenio entre la Universidad de Guadalajara y la Universidad de San Carlos de Guatemala, así como, apoyar diversas acciones de investigación educativa e investigación en Salud Pública. La consultoría en la construcción del Modelo Educativo como un elemento del Desarrollo Curricular de la Facultad de Ciencias Médicas de la USAC, forma parte de este convenio marco.

El aporte del presente reporte de investigación está relacionado con el análisis de las experiencias de internacionalización en el campo de las prácticas curriculares entre dos universidades públicas de la región, en donde existe una tendencia a copiar modelos educativos del primer mundo, frecuentemente ajenos a las culturas educativas de nuestras instituciones educativas.

\section{BASES TEÓRICAS}

La investigación que se analiza se fundamenta en la Teoría Crítica. Desde esta perspectiva epistemológica es importante analizar las transformaciones de los modelos educativos que generan los sujetos sociales de las instituciones académicas, en el contexto de la realidad socioeconómica, histórica, cultural y política a escala local y global. Los criterios de validez metodológica están relacionados epistemológicamente con el potencial de transformación de los datos empíricos, interpretados con las teorías particulares del objeto de estudio. (Mardones y Urzúa, 1994)

Los modelos educativos han evolucionado históricamente de acuerdo a las necesidades sociales y teorías pedagógicas dominantes en cada época. El modelo centrado en el estudiante, se sustenta en la pedagogía construccionista; en el modelo centrado en el profesor, predomina la Pedagogía Conductista. En la época actual, por las nuevas exigencias del contexto, de acercar los procesos educativos a los espacios socio-laborales, se genera el modelo educativo centrado en el desempeño, donde se desarrollan las competencias necesarias y deseadas para desarrollar capacidades para el empleo. (Vélez y Moya, 2019) 


\section{Journal of the Academy $|160|$}

La teoría curricular Crítica (De Alba, 1998) ubica al modelo educativo como una categoría que intermedia las dimensiones externas e internas del currículum universitario; para su construcción es necesario realizar un análisis de las demandas sociales, laborales y profesionales externas para hacerlos pertinentes. Sin embargo, se señala que los modelos educativos en las instituciones deben responder a la sociedad del conocimiento y no sólo a las demandas socio-laborales como se propone en el currículo por competencias (Young et al., 2016; Orozco, 2009). Desde la teoría crítica, el modelo educativo es conceptualizado como una guía teórico-filosófica y pedagógica didáctica para orientar a los profesores en sus prácticas educativas. Es también, una guía conceptual para el desarrollo curricular y la investigación educativa, en donde se definen los aspectos epistemológicos y sociológicos para realizarlas. (Crocker et al., 2009b).

Para dar respuesta a las demandas de pertinencia local y competitividad global, las instituciones educativas universitarias deben integrar en sus procesos sustantivos una estrategia de internacionalización. Para ello, se requiere planificar las organizaciones universitarias desde su propia misión y visión que impacte de desarrollo curricular, que incluye los siguientes elementos: la incorporación de la dimensión internacional y global, el desarrollo de compromisos éticos de los grupos de interés locales e internacionales, el desarrollo del pensamiento complejo, la solidaridad, la transparencia en la acciones que se emprenden y el desarrollo del pensamiento crítico, creativo e innovador en las universidades y la sociedad. (González-Castro y Manzano-Durán, 2015)

Se toma la decisión que la USAC construya su propio paradigma de acuerdo a la realidad intercultural del país, a su propio desarrollo histórico institucional y a los nuevos retos internacionales de la globalización. Se propone el Paradigma Sociohistórico Cultural ${ }^{3}$ propuesto inicialmente por Vygorsky y desarrollados por Leontiev y Rubistein (Anderson, 2013) como el articulador epistémico del nuevo modelo educativo de la USAC. (DIGED USAC, 2018)

\footnotetext{
${ }^{3}$ La Teoría de la actividad humana es una metateoría con raíces en la psicología históricocultural de Lev Vygotsky y posteriormente desarrollados por Leóntiev y Rubinstein, quienes buscaban entender las actividades humanas como complejos fenómenos socialmente situados, e ir más allá de los paradigmas del psicoanálisis y de la psicología conductista.
} 


\section{Journal of the Academy $|161|$}

La premisa central de esta teoría, es que el aprendizaje del individuo no es el único aspecto de análisis. Otras categorías están relacionadas con la historia personal, la clase social y el capital cultural, que también definen las oportunidades sociales que apoyan el aprendizaje y el éxito profesional al egreso.

Desde esta perspectiva epistémica, el aprendizaje es una construcción social del conocimiento que se sitúa en los escenarios reales, es decir, aprendizaje significativo pertinente con lo concreto. El aprendizaje situado en la realidad virtual, implica aprender de forma colaborativa en línea, compartiendo con sus pares, objetivos y tareas, en donde las tecnologías de la información y la comunicación (TICs) son las mediadoras del proceso educativo; para el caso de las universidades se gestiona en el contexto de la sociedad del conocimiento.

Para comprender los paradigmas y su relación con las epistemologías de las Ciencias de la Educación, se presentan los tres paradigmas de la Modernidad Científica y su relación con los modelos educativos: Conductista, Constructivista y Sociocrítico. (Ramírez, 2008; Morales e Irigoyen, 2016; Vélez y Moya, 2019). Se analizan las características ontológicas (concepción del ser humano), sociológicas (relación universidad sociedad), epistemológicas (análisis científico de la realidad), pedagógica (teoría educativa en que se fundamenta) y didáctica (concepción del aprendizaje) de cada uno de los paradigmas. Estos elementos se sistematizan en la Tabla 1.

\section{Tabla 1}

Matriz de análisis de programas de educación con base en las teorías de los modelos educativos

\begin{tabular}{|c|c|c|c|c|c|}
\hline $\begin{array}{c}\text { MODELO } \\
\text { EDUCATIVO }\end{array}$ & $\begin{array}{l}\text { VISIÓN } \\
\text { ONTOLÓGI } \\
\text {-CA } \\
\end{array}$ & $\begin{array}{c}\text { VISIÓN } \\
\text { SOCIOLÓGI } \\
\text {-CA }\end{array}$ & $\begin{array}{c}\text { VISIÓN } \\
\text { EPISTEMOLÓ- } \\
\text { GICA }\end{array}$ & $\begin{array}{l}\text { FUNDAMENTA- } \\
\text { CIÓN } \\
\text { PEDAGÓGICA } \\
\end{array}$ & $\begin{array}{l}\text { ENFOQUE } \\
\text { DIDÁCTICO }\end{array}$ \\
\hline $\begin{array}{c}\text { CONDUCTIS } \\
\text { TA }\end{array}$ & $\begin{array}{l}\text { Ser humano } \\
\text { pasivo al que } \\
\text { se le enseñan } \\
\text { conductas. }\end{array}$ & $\begin{array}{l}\text { La educación } \\
\text { reproduce la } \\
\text { visión de la } \\
\text { estructura } \\
\text { social }\end{array}$ & $\begin{array}{l}\text { Estudia el ser } \\
\text { humano como } \\
\text { objeto } \\
\text { fragmentado. }\end{array}$ & $\begin{array}{l}\text { Aprendizaje de } \\
\text { conductas } \\
\text { previamente } \\
\text { definidas en } \\
\text { objetivos de los } \\
\text { programas por el } \\
\text { educador o } \\
\text { experto. }\end{array}$ & $\begin{array}{l}\text { Se fundamenta en } \\
\text { la enseñanza de } \\
\text { conductas por el } \\
\text { educador a través } \\
\text { de medios e } \\
\text { instrumentos. }\end{array}$ \\
\hline
\end{tabular}




\begin{tabular}{|c|c|c|c|c|c|}
\hline $\begin{array}{c}\text { CONSTRUCT } \\
\text { IVISTA }\end{array}$ & $\begin{array}{l}\text { El ser } \\
\text { humano es } \\
\text { capaz de } \\
\text { construir su } \\
\text { aprendizaje a } \\
\text { través de su } \\
\text { acción } \\
\text { creativa. }\end{array}$ & $\begin{array}{l}\text { El ser humano } \\
\text { actúa } \\
\text { socialmente de } \\
\text { acuerdo a sus } \\
\text { representacion } \\
\text { es. }\end{array}$ & $\begin{array}{l}\text { Se interpreta al } \\
\text { ser humano en } \\
\text { sus } \\
\text { representaciones } \\
\text { subjetivas. }\end{array}$ & $\begin{array}{l}\text { La educación } \\
\text { busca que las } \\
\text { personas } \\
\text { construyan su } \\
\text { aprendizaje con } \\
\text { base en su } \\
\text { representación de } \\
\text { la realidad. }\end{array}$ & $\begin{array}{l}\text { El educador } \\
\text { asesora y apoya a } \\
\text { la persona para } \\
\text { descubrir su } \\
\text { realidad de } \\
\text { acuerdo a su } \\
\text { cultura y } \\
\text { cosmovisión. }\end{array}$ \\
\hline CRÍTICO & $\begin{array}{l}\text { El ser } \\
\text { humano es un } \\
\text { sujeto } \\
\text { sociopolítico } \\
\text { capaz de } \\
\text { comprender y } \\
\text { transformar } \\
\text { su realidad. }\end{array}$ & $\begin{array}{l}\text { La educacion } \\
\text { busca educar a } \\
\text { sujetos para la } \\
\text { acción crítica } \\
\text { del contexto } \\
\text { social. }\end{array}$ & $\begin{array}{l}\text { Se analiza al ser } \\
\text { humano y a las } \\
\text { instituciones en } \\
\text { su contexto } \\
\text { social, } \\
\text { económico y } \\
\text { político. }\end{array}$ & $\begin{array}{l}\text { La educación } \\
\text { forma a las } \\
\text { personas para la } \\
\text { liberación personal } \\
\text { y la acción } \\
\text { transformadora de } \\
\text { la sociedad. }\end{array}$ & $\begin{array}{l}\text { El educador se } \\
\text { incorpora a la } \\
\text { realidad de los } \\
\text { sujetos sociales } \\
\text { para realizar un } \\
\text { proceso educativo } \\
\text { transformador de } \\
\text { tipo participativo. }\end{array}$ \\
\hline
\end{tabular}

Fuente: Elaboración propia con base en criterios de: Ramírez (2008); Morales e Irigoyen (2016); Vélez y Moya (2019)

\section{METODOLOGÍA}

En el proceso de investigación educativa para la construcción del Modelo Educativo de la USAC, como parte del Programa de Desarrollo Curricular de la Facultad de Ciencias Médicas, asesorada por el coordinador de la investigación curricular, como miembro de la Universidad de Guadalajara, en el contexto del Programa de Internacionalización Universitaria, participaron los siguientes actores sociales: un representante de Rectoría de la USAC, el Director Académico y 3 pedagogos de la DIGED, 3 representantes por cada una de las unidades académicas y programas educativos de la USAC (44 académicos en total), con formaciones diversas: pedagogos, sociólogos, administradores educativos, médicos, psicólogos, odontólogos, arquitectos, ingenieros, agrónomos, veterinarios, economistas, trabajadoras sociales, entre otras profesiones universitarias. Los académicos fueron distribuidos en 4 equipos de trabajo para construir las cuatro dimensiones del Modelo Educativo: filosófica, administrativo-académica, pedagógica didáctica y gestión de la calidad educativa.

La metodología seguida para la implementación de la estrategia para construir el Modelo Educativo como parte del desarrollo curricular de la Facultad de Ciencias Médicas, en particular y de la USAC en general, así como la acción de internacionalización del currículo por la Universidad de Guadalajara, se fundamenta en un diseño de Investigación Acción Participativa. 


\section{Journal of the Academy $|163|$}

Las razones por las cuales el equipo coordinador de la DIGED de la USAC, los coordinadores de la Facultad de Ciencias Médicas y el consultor de la Universidad de Guadalajara, toman la decisión de utilizar la Metodología de Investigación Acción Participativa son las siguientes:

La Investigación Acción Participativa (IAP) como un tipo investigación cualitativa participante, aunque se puede triangular con investigación cuantitativa, es apropiada para propiciar procesos de transformación e internacionalización curricular, como el que se presenta. Algunos autores, señalan que este método expande el conocimiento científico y la solución de un problema, así como, desarrolla las capacidades de los respectivos participantes en los procesos de transformación. (Martínez, 1991)

La IAP, es un diseño metodológico que se utiliza para obtener conocimientos sobre una determinada realidad colectivamente. Es una actividad integral con las siguientes fases: la investigación social, la educación de los participantes, la acción transformadora y la evaluación del proceso. Como parte de la IAP realizada en la USAC, un proceso pendiente es la implementación del modelo educativo, aspecto que será realizado por las unidades académicas en la reforma de sus planes de estudio, así como la evaluación del modelo, que debe ser desarrollada en el proceso para acreditar la calidad de los programas académicos de la USAC.

El problema que se aborda, el modelo educativo como parte del desarrollo curricular, se origina en la propia comunidad universitaria de la USAC; el objetivo de la investigación es la transformación universitaria a partir del modelo educativo, el cual debe repercutir en la mejora de la calidad educativa. En el proceso de IAP realizado, los beneficiarios de la transformación son los profesores, alumnos e investigadores de la institución, quienes de manera participativa han realizado y conducido el proceso de investigación, lo que fortalece y potencializa la conciencia crítica de los sujetos sociales sobre sus propias debilidades, habilidades y recursos y los moviliza como investigadores de su propia realidad. (Martínez, 1991)

Para recabar los datos del diagnóstico curricular y la discusión de los elementos que forman parte de las categorías del Modelo Educativo de la USAC, se utilizan las siguientes técnicas: análisis del discurso documental, entrevistas en profundidad de informantes clave, los talleres de diálogo en los equipos de trabajo y las relatorías de las plenarias de presentación de datos para elaborar conclusiones y recomendaciones. 


\section{Journal of the Academy $|164|$}

Para sistematizar los datos empíricos recolectados a lo largo del proceso de investigación acción participativa y poder construir las dimensiones y categorías del modelo educativo, se utiliza la matriz de análisis que se presenta en la tabla siguiente:

\section{Tabla 2}

Procedimientos de análisis de datos

\begin{tabular}{|c|c|c|c|c|}
\hline $\begin{array}{l}\text { Datos } \\
\text { empíricos }\end{array}$ & Codificación & $\begin{array}{l}\text { Unidades de } \\
\text { análisis }\end{array}$ & $\begin{array}{l}\text { Interpretación } \\
\text { empírico-teórica }\end{array}$ & $\begin{array}{l}\text { Construcción } \\
\text { ensayo } \\
\text { dimensión }\end{array}$ \\
\hline $\begin{array}{l}\text { Narraciones del } \\
\text { texto, del } \\
\text { informante } \quad y \\
\text { memorias del } \\
\text { taller }\end{array}$ & $\begin{array}{l}\text { Conceptos } \\
\text { centrales que se } \\
\text { ubican en los } \\
\text { datos }\end{array}$ & $\begin{array}{l}\text { Ubicación de } \\
\text { los conceptos } \\
\text { en la unidad de } \\
\text { análisis del } \\
\text { mapa } \\
\text { conceptual }\end{array}$ & $\begin{array}{l}\text { Análisis de los datos } \\
\text { empíricos y } \\
\text { conceptos por } \\
\text { unidad de análisis } \\
\text { de acuerdo a los } \\
\text { teóricos definidos y } \\
\text { otros que sean } \\
\text { necesarios }\end{array}$ & $\begin{array}{l}\text { Introducción, } \\
\text { desarrollo } \\
\text { ensayo, } \\
\text { conclusiones, } \\
\text { referencias } \\
\text { bibliográficas } \\
\text { (APA) }\end{array}$ \\
\hline
\end{tabular}

Fuente: Elaboración propia (USAC, 2018)

\section{RESULTADOS}

Los resultados generados por la estrategia para construir el modelo educativo, como parte de la internacionalización del desarrollo curricular universitario con el Enfoque de Competencias Profesionales Integradas, son agrupados por los autores del presente artículo, que son parte del proceso de investigación acción participativa, en tres partes: en la primera, se presentan las bases teóricas del proceso formativo de los participantes en la investigación para realizar el diagnóstico y el diseño del Modelo Educativo, que fueron reflexionadas con las bases teóricas de la presente investigación; en la segunda, se analizan los resultados del diagnóstico participativo y en la tercera, se presentan los elementos centrales que caracterizan el Modelo Educativa de la USAC. 


\section{Journal of the Academy $|165|$}

\section{Diagnóstico de la problemática de desarrollo curricular a resolver por el Modelo Educativo de la USAC}

En el diagnóstico de la problemática de desarrollo curricular a resolver por el modelo educativo de la USAC se analiza el contexto externo, en donde se identifica una sociedad en transición, con predominio de exclusión social, polarizada entre las demandas del Estado y la Sociedad Civil, con escasa competitividad económica en un contexto global complejo. De ese análisis se deriva la propuesta de construir un modelo educativo, en donde se medien las propuestas de pertinencia y competitividad a escala local y global.

Se realiza un análisis de la dimensión interna del currículum, en donde se identifican problemas relacionados con deficiencias en las competencias transversales de los alumnos, planes de estudios disciplinares rígidos, aprendizaje teorizante, así como, un divorcio entre el discurso de los diseños curriculares y la práctica educativa que realizan los docentes, por lo que se propone realizar procesos de planeación educativa participativos, que acerquen a los docentes y alumnos a los escenarios socio-laborales

Otros problemas que se analizan en la dimensión interna del currículum, son la escasa investigación educativa y deficiencias en la cultura de calidad, así como, una administración académica rígida, divorciada de los escenarios reales para aplicar modelos educativos por competencias, por lo que se proponen alternativas para impulsar la investigación educativa de los problemas socio-laborales, como parte de un programa de desarrollo curricular, así como, programas de aseguramiento de la calidad que transformen la gestión administrativo académica.

Un elemento importante detectado en el diagnóstico, son los problemas en la formación docente, que está alejada de los procesos de transformación hacia modelos por competencias, por lo que sugieren reforzar esta estrategia en el modelo educativo.

\section{Elementos de la propuesta de modelo educativo de la USAC}

Al analizar los elementos del modelo educativo construido en los talleres realizados en la USAC, se identifican cuatro dimensiones: Filosófica, Académico-administrativa, pedagógica- 


\section{Journal of the Academy $|166|$}

didáctica y Gestión de Calidad. Las tres primeras dimensiones se retoman, después de un análisis crítico, de la experiencia del modelo educativo del Centro Universitario de Ciencias de la Salud de la Universidad de Guadalajara (Crocker et al., 2009a) La última dimensión, es una propuesta innovadora del modelo de la USAC, como un elemento que da respuesta a las demandas regionales y globales de aseguramiento de la calidad en las universidades.

En la Tabla 3 se presenta una síntesis esquemática de los elementos centrales de las dimensiones del modelo educativo de la USAC. La dimensión filosófica está constituida por las visiones ontológica, axioteleológica, epistemológica y sociológica de la propuesta educativa universitaria; en la dimensión académico administrativa se presentan los referentes curriculares de los planes y programas de estudio y los elementos para realizar la operación de las funciones sustantivas relacionadas con docencia, investigación, vinculación y difusión; en la dimensión pedagógico-didáctica, se caracteriza la teoría que sustenta la práctica educativa, los procesos de enseñanza aprendizaje y los medios y recursos didácticos para su implementación; por último, se presentan los elementos de la dimensión de gestión de calidad que orientan la promoción de la mejora continua de las funciones sustantivas universitarias en dirección de promover una cultura de calidad y la acreditación externa de sus programas educativos.

Los elementos del Modelo Educativo (USAC, 2018) con una extensión de ochenta páginas, en donde se generan créditos para los 44 participantes, el equipo de Coordinación y al Consultor de la Universidad de Guadalajara, coordinador del presente estudio, son presentados a la DIGED y posteriormente al Consejo Superior Universitario de la USAC, para su aprobación e implementación en las áreas académicas de la institución.

\section{Tabla 3}

Elementos de las dimensiones del Modelo educativo de la USAC

\begin{tabular}{|c|c|c|c|c|c|c|c|c|}
\hline $\begin{array}{l}\text { DIMEN } \\
\text { SIONES }\end{array}$ & FILOSÓ1 & ICA & & $\begin{array}{l}\text { PEDAC } \\
\text { DIDÁC }\end{array}$ & $\begin{array}{l}\text { ICO- } \\
\mathrm{A}\end{array}$ & $\begin{array}{l}\text { ACADÉM } \\
\text { ADMINIS }\end{array}$ & $\begin{array}{l}\text { O } \\
\text { RATIVA }\end{array}$ & $\begin{array}{l}\text { EVALUACI } \\
\text { ÓN DE } \\
\text { CALIDAD }\end{array}$ \\
\hline $\begin{array}{l}\text { CATEG } \\
\text { ORÍAS }\end{array}$ & $\begin{array}{l}\text { Ontológi } \\
\text { ca }\end{array}$ & $\begin{array}{l}\text { Episte- } \\
\text { mológi- } \\
\text { ca }\end{array}$ & $\begin{array}{l}\text { Socio- } \\
\text { lógica }\end{array}$ & $\begin{array}{l}\text { Peda- } \\
\text { gógica }\end{array}$ & Didáctica & $\begin{array}{l}\text { Curricula } \\
\mathbf{r}\end{array}$ & $\begin{array}{l}\text { Gestión } \\
\text { académi- } \\
\text { ca }\end{array}$ & $\begin{array}{l}\text { Gestión de } \\
\text { calidad }\end{array}$ \\
\hline
\end{tabular}


Journal of the Academy | 167|

\begin{tabular}{|c|c|c|c|c|c|c|c|c|}
\hline $\begin{array}{l}\text { CARAC } \\
\text { TERÍST } \\
\text { ICAS }\end{array}$ & $\begin{array}{l}\text { Sujeto- } \\
\text { sociohist } \\
\text { órico } \\
\text { cultural } \\
\text { que } \\
\text { construye } \\
\text { su visión } \\
\text { del } \\
\text { mundo } \\
\text { con } \\
\text { libertad } \\
\text { en } \\
\text { contextos } \\
\text { esttructu- } \\
\text { rados y } \\
\text { comple- } \\
\text { jos }\end{array}$ & $\begin{array}{l}\text { Gestión y } \\
\text { transfere } \\
\text { ncia } \\
\text { social del } \\
\text { conocimi } \\
\text { ento con } \\
\text { enfoque } \\
\text { complejo } \\
\text { transdisci } \\
\text { plinar }\end{array}$ & $\begin{array}{l}\text { Relación } \\
\text { crítica } \\
\text { con la } \\
\text { realidad } \\
\text { social y } \\
\text { laboral } \\
\text { que } \\
\text { promuev } \\
\text { e la } \\
\text { inclusión } \\
\text { la } \\
\text { equidad } \\
\text { y el } \\
\text { diálogo } \\
\text { de } \\
\text { saberes }\end{array}$ & $\begin{array}{l}\text { Promueve } \\
\text { las } \\
\text { competen } \\
\text { cias de la } \\
\text { persona } \\
\text { para } \\
\text { analizar y } \\
\text { resolver } \\
\text { problema } \\
\text { s en } \\
\text { equipos } \\
\text { intercultu } \\
\text { rales }\end{array}$ & $\begin{array}{l}\text { Profesor y } \\
\text { alumno } \\
\text { analizan la } \\
\text { realidad } \\
\text { sociolabora } \\
\text { l y } \\
\text { gestionan } \\
\text { el } \\
\text { conocimie } \\
\text { nto con } \\
\text { enfoque } \\
\text { complejo }\end{array}$ & $\begin{array}{l}\text { Diseños } \\
\text { curriculare } \\
\text { s } \\
\text { semiflexibl } \\
\text { es por } \\
\text { competenci } \\
\text { as } \\
\text { profesional } \\
\text { es } \\
\text { integradas } \\
\text { con } \\
\text { pertinencia } \\
\text { sociolabora } \\
1\end{array}$ & $\begin{array}{l}\text { Gestión } \\
\text { estratégica } \\
\text { y operativa } \\
\text { participativ } \\
\text { a que } \\
\text { integra } \\
\text { docencia, } \\
\text { investigaci } \\
\text { ón y } \\
\text { extensión }\end{array}$ & $\begin{array}{l}\text { Promueve la } \\
\text { responsabilid } \\
\text { ad social } \\
\text { para el } \\
\text { asegura- } \\
\text { miento de la } \\
\text { calidad } \\
\text { interna y } \\
\text { externa, la } \\
\text { pertinencia y } \\
\text { la } \\
\text { competitivid } \\
\text { ad. }\end{array}$ \\
\hline
\end{tabular}

Fuente: Elaboración propia (USAC, 2018)

En la dimensión filosófica del modelo educativo se propone una visión ontológica, en donde se concibe al ser humano que actúa con libertad en contextos complejos estructurados por la globalización; investiga con epistemologías sustentadas en el pensamiento complejo, la realidad socio-laboral en equipos transdisciplinares y fomenta los valores de equidad e interculturalidad en una sociedad excluyente, como la realidad social guatemalteca.

En la dimensión pedagógico-didáctica, se proponen las competencias profesionales integradas para analizar y resolver problemas en equipos interculturales con participación activa de profesores y alumnos que analizan con pensamiento crítico el conocimiento producido en la sociedad global compleja y lo validan para recrearlo en la realidad socio-laboral de Guatemala. En la dimensión administrativo-académica, se propone un desarrollo curricular para construir planes y programas de estudio semiflexibles con pertinencia social, profesional y laboral, los que se deben construir con el enfoque de Competencias Profesionales Integradas, en donde se incorpore la gestión integrada de las funciones sustantivas de docencia, investigación y servicio.

Por último, se propone incorporar la dimensión de gestión de calidad en todos los procesos académicos y administrativos relacionados con las funciones sustantivas de docencia, investigación y servicio, para propiciar su aseguramiento interno y la acreditación ante organismos externos. 


\section{Journal of the Academy $|168|$}

La experiencia de internacionalización curricular para la construcción del Modelo Educativo de la USAC, en el contexto de las demandas de acreditación de calidad de los programas educativos de las universidades a escala global, aporta elementos teórico- metodológicos para la realidad latinoamericana, con énfasis en el enfoque de competencias profesionales integradas y en la metodología de Investigación Acción Participativa.

El modelo educativo es conceptualizado como una guía teórico-filosófica y pedagógica didáctica para orientar a los profesores en sus prácticas educativas. Es también, una guía conceptual para el desarrollo curricular y la investigación educativa en donde se definen los aspectos epistemológicos y sociológicos para realizarlas. (Crocker et al., 2009a)

En el campo de la internacionalización curricular, con énfasis en modelos educativos a nivel latinoamericano, es importante recuperar la percepción de los docentes en los procesos de transformación. Al respecto, el estudio de las concepciones culturales de los docentes sobre el modelo educativo de una universidad pública de Ecuador realizada por investigadores del Doctorado de Salud Ocupacional del CUCS (García et al., 2020) se señala, la importancia del Modelo Educativo en el desarrollo curricular de las instituciones educativas en donde se destacan los siguientes aspectos: a) la importancia de contar con nuevos enfoques pedagógicos y aproximaciones metodológicas con perspectiva crítico-reflexiva; b) La importancia de analizar las transformaciones sociales y del conocimiento, a través de incorporar estrategias didácticas con el uso de nuevas tecnologías de la información y de la comunicación.

Uno de los problemas que se identifican para la implementación del nuevo modelo educativo de la USAC es la escasa formación de líderes que promuevan el cambio. En el ámbito académico, se recomienda propiciar liderazgos que fomenten la transformación universitaria, a través de generar procesos de la gestión participativa sociopolítica de los actores académicos, que, para el caso de Guatemala, contribuyan a desarrollar un país incluyente, con equidad social, étnica y de género con competitividad en el contexto global. (Crocker y Veloso, 2019)

En la transformación del modelo educativo de la USAC un aspecto importante es la decisión tomada, con relación a fundamentarla en el paradigma Socio-histórico Cultural. La propuesta es pertinente con la institución, que tiene una historia y liderazgo en las transformaciones de la 


\section{Journal of the Academy $|169|$}

educación universitaria y en el desarrollo de la cultura en el contexto de una sociedad guatemalteca multiétnica, multicultural y plurilingüe.

En esa dirección, una primera tarea metodológica fue revisar los antecedentes para interpretar el discurso institucional, para comprender su evolución y cuáles son las tesis centrales relacionadas con lo educativo. Con base en esta recomendación y en coherencia con el Paradigma Socio-histórico Cultural los equipos de trabajo revisan los siguientes documentos externos e internos, que constituyen elementos para construir el Modelo Educativo de la USAC: Constitución de la República de Guatemala de 1984, relacionado con el marco legal de la educación superior; el documento de Reforma Universitaria de la USAC de 1986; el marco filosófico de la USAC de Junio 1998; los documentos del Consejo Superior Universitario relacionados con el marco académico de la USAC y el marco de la realidad y necesidades de la sociedad Guatemalteca de1998, así como el Plan estratégico de la USAC 2002, 2003 y la Visión de la USAC del 2013.

Como apoyo educativo y cultural al trabajo de los equipos de elaboración del Modelo Educativo, se analiza: el Marco Estratégico de la Dirección de Desarrollo Educativo (DIGED) del 2017, las Guías de Evaluación de la Educación Superior del Consejo Superior de Universidades de Centro América (CSUCA) del 2015 y el Libro Azul de la USAC, relacionado con políticas de educación ambiental, de la mujer e inclusión social elaborado en el año 2015.

\section{CONCLUSIONES}

A continuación, se presentan las características identificadas como determinantes para los buenos resultados de la estrategia de internacionalización conducida por los autores en la construcción del Modelo Educativo de la USAC, como un elemento del desarrollo curricular. Para la construcción del Modelo Educativo de la USAC, ha sido importante la experiencia sistematizada por un grupo de investigadores en educación de la Universidad de Guadalajara. Los aportes para construir la propuesta desde una perspectiva innovadora de la educación superior con el Enfoque de Competencias Profesionales Integradas, a partir de la experiencia consolidada desde el año 2006. 


\section{Journal of the Academy $|170|$}

Una buena práctica en la internacionalización del desarrollo curricular es la gestión social que se realiza con las universidades con quienes se establece vinculación, como parte de un programa de largo plazo. Las acciones aisladas no generan transformaciones y no se puede recuperar experiencias valiosas. El proyecto de construcción del Modelo Educativo de la USAC, tiene como antecedente la firma de un convenio de colaboración internacional desde el año 2005, que ha incluido vinculaciones previas en asesoría curricular en el campo de las Ciencias Médicas, investigación de servicios de salud pública, intercambio de profesores, investigadores y alumnos entre ambas instituciones.

Una buena práctica de internacionalización para el desarrollo curricular en instituciones de educación superior, con quienes se realiza vinculación universitaria es la instrumentación de la metodología de investigación acción participativa. La IAP, es un diseño metodológico que se utiliza para obtener conocimientos sobre una determinada realidad colectivamente, es una actividad integral que combina la investigación social, la educación de los participantes, la acción transformadora, la implementación y la evaluación y para el caso particular de la investigación, los procesos de internacionalización curricular.

La práctica de internacionalización para construir el Modelo Educativo de la USAC, como un elemento para el Desarrollo Curricular de esta institución educativa en Centro América, genera dos aspectos valiosos para las Ciencias de la Educación: la propuesta epistemológica Sociohistórica Cultural en contextos complejos, con el que viven las instituciones de educación superior públicas en América Latina; la incorporación de la dimensión de Gestión de Calidad, como parte de los modelos educativos, debido a la demanda internacional de acreditación de las propuestas curriculares de las instituciones universitarias.

La construcción del modelo educativo en las instituciones universitarias debe ser una estrategia fundamental como parte de los programas de desarrollo curricular, ya que constituye el marco filosófico, pedagógico-didáctico y de gestión para asegurar la calidad de las funciones sustantivas de docencia, investigación y vinculación que guía las acciones de profesores, alumnos, investigadores y administradores de las universidades.

La construcción, implementación y evaluación del modelo educativo de las universidades debe estar sustentado en un programa de investigación educativa transdisciplinar, con líneas de investigación, en donde se analicen las dimensiones filosóficas, sociológica, pedagógico- 


\section{Journal of the Academy $|171|$}

didáctica, administrativo y de aseguramiento de la calidad interna en contextos complejos, que permitan que las instituciones educativas sean competitivas a escala global. En el proceso es importante la asesoría y apoyo de grupos de investigadores con experiencia en gestión del conocimiento de frontera del campo de las ciencias sociales y humanas, así como, la publicación en revistas especializadas que permitan generar redes para compartir los hallazgos y teorías que fortalezcan el campo teórico del currículum universitario.

\section{REFERENCIAS BIBLIOGRÁFICAS}

Anderson, G. (2013). The Activity Theory Approach - El enfoque de la Teoría de la Actividad. Capítulo libro prelanzamiento.

Crocker, R. Hunot, C. González, M. Cuevas, L. y Vizmanos, B. (2008). Gestión académica del curriculum por competencias. El proceso de construcción social con las licenciaturas en Nutrición de la Universidad de Guadalajara. Universidad de Guadalajara, Centro Universitario de Ciencias de la Salud

Crocker, R. Farfán, P. Huerta, J. Cuevas, L. y González, M. (2009a). Modelo Educativo del Centro Universitario de Ciencias de la Salud. Universidad de Guadalajara, Centro Universitario de Ciencias de la Salud.

Crocker, R. Cuevas, L. Hunot, C. González, M. López, P. (2009b). Desarrollo Curricular por Competencias Profesionales Integradas. La experiencia del Centro Universitario de Ciencias de la Salud de la Universidad de Guadalajara (2 ${ }^{d a}$ ed.). Universidad de Guadalajara.

Crocker, R. y Veloso, A. (2019). Nuevos enfoques de liderazgo para la innovación de la educación médica en México. En: Díaz, M. y Veloso, A. (eds) Modelos de Educación en Liderazgo Educativo. Una revisión internacional. UNAM.

De Alba, A. (1998) Curriculum: crisis, mitos y perspectivas. Miño y Dávila editores S. R. L. www.terras.edu.ar/biblioteca/1/CRRM_De_Alba_Unidad_1.pdf

Dirección General de Desarrollo Académico de la Universidad de San Carlos de Guatemala. (2018). Modelo Educativo de la Universidad de San Carlos de Guatemala. DIGED/USAC.

Farfán, P., Pérez, I., Matsui, O., Crocker, R. y López, A. (2010). Competencias Profesionales Integradas. Evaluación y rediseño Curricular. Universidad de Guadalajara.

García, M., Contreras, M., Mercado, M., Saravia, M. y León, S. (2020). Concepciones culturales de los docentes sobre el modelo educativo en una Universidad Pública de Ecuador. Revista Educación y desarrollo, 28 (2) 81-102. http://www.dspace.uce.edu.ec/handle/25000/22584 
González-Castro, y Manzano-Durán, O. (2015). La internacionalización curricular desde las competencias de la responsabilidad social universitaria. Libre Empresa, 12(2), 27-38 http://dx.doi.org/10.18041/libemp.2015.v12n2.24202

Mardones, J. M. y Urzúa, N. (1994). Filosofía de las ciencias humanas y sociales. Editorial Fontamara, S. A

Martínez, J. (2005). Calidad de la Educación Superior en la Región Centroamericana. DIGED.

Martínez, M. (1991). La investigación Cualitativa Etnográfica en Educación. Litexsa Venezolana

Morales, H. e Irigoyen, A. (2016). Paradigma Conductista y Constructivista de la Educación a través del Decálogo del Estudiante. Archivos de Medicina Familiar, 18 (2), 27-30. https://psicologiaymente.com/psicologia/conductismo-constructivismo-

Orozco, B. (2009) Competencias y currículum: una relación tensa y compleja. RISEU http://www.riseu.unam.mx/documentos/acervo_documental/txtid0057.pdf

Ramírez, R. (2008) La pedagogía crítica Una manera ética de generar procesos educativos. $\begin{array}{lllll}\text { Revista Folios } & \text { Segunda } & \text { (28) } & \text { 108-119. }\end{array}$ https://www.scielo.org.co/pdf/folios/n28/n28a09.pdf

Universidad de Guadalajara/USAC. (2005). Convenio de Vinculación Académica Universidad de Guadalajara con la Universidad de San Carlos de Guatemala. Universitaria.

Universidad de San Carlos de Guatemala (2018). Modelo Educativo de la Universidad de San Carlos de Guatemala. USAC.

Vélez, J. y Moya, M (2019). Los modelos educativos y su relación con las teorías del aprendizaje. Atlante. Cuadernos de Educación y Desarrollo. https://www.eumed.net/rev/atlante/2019/08/modelos-educativos-aprendizaje.html

Young, M., Parra, G. y Macias, L. (2016). El futuro de la educación en una sociedad del conocimiento: el argumento radical en defensa de un currículo centrado en materias. Pedagogía y saberes, 45, 79-88. https://doi.org/10.17227/01212494.45pys79.88 